\title{
Avaliação da pressão arterial no ambulatório Revisão baseada na evidência
}

Hélder Aguiar, ${ }^{*}$ Ana Isabel Silva, ${ }^{* *}$ Fernando Pinto, ${ }^{* * *}$ Susana Catarino****

\section{RESUMO}

Objectivos: Determinar: a evidência da utilidade da auto-medição da pressão arterial (AMPA) e da monitorização ambulatória da pressão arterial de 24h (MAPA) como parte integrante do processo de avaliação e seguimento dos indivíduos hipertensos, em três domínios-chave - risco cardiovascular, controlo pressão arterial (PA) e relação custo-benefício - quando comparadas com o uso isolado da PA medida no consultório; o valor prognóstico da PA diurna e nocturna, e a sua relação, obtidas através da MAPA.

Fontes de dados: Bases de dados Medline e de Medicina Baseada na Evidência.

Métodos de revisão: Pesquisa de normas de orientação clínica (NOC), revisões sistemáticas, meta-análises, ensaios clínicos aleatorizados (ECA) e estudos observacionais, publicados entre Janeiro de 2000 e Maio de 2011, utillizando o termo MeSH «blood pressure monitoring, ambulatory» e os termos «home blood pressure monitoring» e «self blood pressure monitoring». Foi aplicada a escala SORT da American Family Physician para classificar a evidência.

Conclusões: Foram seleccionados 38 artigos: quatro NOC, nove meta-análises, duas revisões sistemáticas, 19 ECA e quatro estudos observacionais. A inclusão de cada uma das técnicas do ambulatório permite: reduzir o efeito da bata branca, obtendo correlação mais significativa com os eventos cardiovasculares e maiores taxas de controlo tensional; reduzir os custos por via da redução de fármacos e dias de tratamento.

Para cada uma das técnicas de ambulatório estudadas, há evidência de que a sua utilização pode fornecer uma avaliação diagnóstica e prognóstica mais fidedignas (força de recomendação A para a MAPA e força de recomendação B para a AMPA). Estas técnicas podem resultar em melhor controlo da PA no hipertenso (força de recomendação A para MAPA e AMPA). A medição ambulatorial pode também reduzir os custos (força de recomendação B para MAPA e AMPA).

Palavras-chave: Hipertensão; Monitorização Ambulatorial da Pressão Arterial; Auto-Medição da Pressão Arterial.

\section{INTRODUÇÃO}

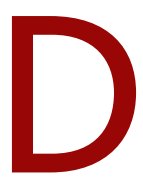

escrita há mais de um século por Koroktoff, a técnica clássica de medição do consultório tem persistido como o método de eleição para a avaliação da pressão arterial (PA). No entanto, nas últimas décadas, a literatura tem vindo a identificar nesta várias limitações. ${ }^{1-4}$ Estas derivam sobretudo do facto de se aplicar um método de medi-

\footnotetext{
*Interno de Medicina Geral e Familiar, USF Vale do Vouga (S. João da Madeira), ACES Entre Douro e Vouga II - Aveiro Norte.

**Interna de Medicina Geral e Familiar, USF Nova Salus (V.N.Gaia), ACES Grande Porto VIII - Gaia.

***Assistente Graduado de Cardiologia, Serviço de Cardiologia do Centro Hospitalar Entre Douro e Vouga.

****Assistente de Medicina Geral e Familiar, USF Vale do Vouga (S. João da Madeira), ACES Entre Douro e Vouga II - Aveiro Norte.
}

ção casual e temporalmente pontual - a medição no consultório - a um parâmetro com uma variabilidade fisiológica considerável ao longo das 24 h diárias. ${ }^{5}$

Desta simbiose podem resultar dois erros grosseiros de avaliação diagnóstica. Por um lado, podem ser diagnosticados como hipertensos indivíduos com médias diárias normais. Esta situação - hipertensão da bata branca - pode encontrar-se em percentagens superiores a $20 \% .^{2}$ Por outro lado, os indivíduos hipertensos não apresentam os valores de PA sempre acima do limiar diagnóstico ao longo das $24 \mathrm{~h}$ diárias, podendo originar o fenómeno da ocultação diagnóstica - hipertensão mascarada-descrita recentemente por Thomas Pickering. $^{3}$

Os prejuízos para os doentes podem ser significativos. Considerando as duas situações, mais de um terço 
dos utentes da nossa prática clínica podem estar diagnosticados erradamente, uma parte deles crendo ter um problema de saúde na realidade inexistente e fazendo medicação sem necessidade - na hipertensão da bata branca - e outra parte perpetuando um risco vascular elevado - na hipertensão mascarada. Outra questão suplementar se poderá ainda levantar. Considerando as falhas metodológicas do método convencional do consultório, será este suficientemente preciso para a avaliação no seguimento dos hipertensos efectivos?

Uma correcta medição e interpretação da PA é essencial para o diagnóstico e avaliação da HTA, um factor de risco com um impacto importante no indivíduo e na sociedade. ${ }^{1,6,7}$ A medição da PA no ambulatório teoricamente confere vantagens porque, ao contrário da medição casual do consultório, é realizada num ambiente neutro para o utente, permitindo ainda retirar informação de uma sequência temporal. ${ }^{1}$

A auto-medição da pressão arterial (AMPA) e a monitorização ambulatória da pressão arterial de $24 \mathrm{~h}$ (MAPA) são as duas grandes técnicas de medição da PA no ambulatório. ${ }^{1}$ A AMPA obtém a sequência temporal da PA através da média de várias medições - segundo a Sociedade Europeia de Hipertensão, ${ }^{8}$ a média de sete dias de medições, pelo menos duas de manhã e duas à noite, desprezando os resultados do primeiro dia. A MAPA obtém a sequência nas $24 \mathrm{~h}$ diárias. ${ }^{9}$ Permite ainda obter informações adicionais, nas quais se destaca o perfil circadiano, o qual classifica o hipertenso como dipper, não dippere reverse dipper, consoante o grau de descida de PA nocturna em relação à diurna. ${ }^{5}$

As técnicas de medição da PA no ambulatório têm vindo a registar evidência crescente nas últimas décadas. ${ }^{10}$ Desde $1983^{11}$ que a MAPA tem vindo a ser sustentada como técnica muito vantajosa em termos prognósticos, qualidade que lhe é apontada por permitir extrair informação das $24 \mathrm{~h}$ diárias. Dentro da MAPA, a relação entre PA diurna e nocturna, estratificando os doentes em dippers, non-dippers e reverse dippers, tem sido considerada um excelente marcador prognóstico. $^{2,9}$ Foi em 1988 que O'Brien ${ }^{12}$ reportou pela primeira vez a associação entre um ritmo circadiano anormal, traduzido por descida menos acentuada da PA nocturna - perfil non-dipper - e acidentes vasculares cerebrais. Já a AMPA, como técnica validada para o estudo do doente hipertenso, teve um desenvolvimento mais recente, aliada ao potencial criado pela proliferação dos aparelhos de auto-medição. ${ }^{1,4}$

A conveniência da utilização da técnica convencional do consultório, bem como a confiança inerente a décadas de utilização isolada deste método, têm dificultado uma aplicação mais generalizada das técnicas do ambulatório. Por outro lado, existe ainda um desconhecimento da evidência em relação à aplicabilidade destas técnicas.

Este artigo de revisão baseada na evidência (RBE) pretende explorar a evidência da utilidade de cada uma das técnicas de medição da PA do ambulatório como parte integrante da avaliação e seguimento do doente hipertenso. Para responder a esta questão complexa, estas técnicas foram comparadas com a técnica convencional do consultório em três domínios-chave: capacidade de identificação do risco cardiovascular (CV), avaliação e controlo no seguimento do doente hipertenso e relação custo-benefício.

\section{MÉTODOS}

Foi realizada uma pesquisa bibliográfica de artigos publicados desde Janeiro de 2000 até Maio de 2011 nas seguintes bases de dados: Pubmed, Cochrane, National Guideline Clearinghouse, Trip Database, DARE, Bandolier, Índex de Revistas Médicas Portuguesas e referências bibliográficas dos artigos seleccionados. Foram pesquisadas normas de orientação clínica, meta-análises, revisões sistemáticas, ensaios clínicos (ECA) e estudos observacionais. Foi realizada pesquisa suplementar nas quatro revistas de dedicação exclusiva à HTA com maior factor de impacto internacional: Hypertension, Journal of Hypertension, American Journal of Hypertension e Journal of Human Hypertension. A pesquisa inicial foi realizada entre 6 de Fevereiro a 30 de Junho de 2010. Após o artigo ter sido aceite para publicação nesta revista, foi realizada pesquisa suplementar entre 1 a 5 de Junho de 2011, para actualização da revisão com artigos publicados entre Julho de 2010 a Maio de 2011. Foram utilizadas, nos motores de busca, as seguintes palavras-chave: o termo MeSH «ambulatory blood pressure monitoring», bem como os termos «home blood pressure monitoring» e «self blood pressure monitoring» e os equivalentes na língua portuguesa. Foi decidida a utilização dos dois últimos termos com o intuito de diminuir a exclusão potencial de artigos relevantes relativos à AMPA, uma vez 
que esta técnica não dispõe de um termo MeSH exclusivo. Foram seleccionados artigos relevantes para o objectivo proposto nas línguas inglesa e portuguesa, e foram excluídos os artigos centrados na abordagem em crianças e grávidas. A selecção final foi realizada atendendo à qualidade dos aspectos metodológicos. Foi aplicada a escala Strength of Recommendation Taxonomy $(\mathrm{SORT})^{13}$ da American Family Physician para classificar em níveis de evidência e forças de recomendação. Segundo esta taxonomia, a qualidade do estudo está subdividida em três Níveis de Evidência (Nível de Evidência 1: estudos de boa qualidade, evidência orientada para o doente; Nível de Evidência 2: estudos de qualidade limitada, evidência orientada para o doente; Nível de Evidência 3: outra evidência) e a Força de Recomendação em três graus (Força de Recomendação A: consistente, evidência orientada para o doente; Força de Recomendação B: inconsistente ou qualidade limitada, evidência orientada para o doente; e Força de Recomendação C: consensos, evidência orientada para a doença).

\section{RESULTADOS}

Foram encontrados 6522 estudos, tendo sido seleccionados para esta revisão 38 artigos: duas revisões sistemáticas, nove meta-análises, quatro normas de orientação clínica, 19 ECA e quatro estudos observacionais. Os restantes foram excluídos por divergirem do objectivo do trabalho, por não cumprirem os critérios de inclusão ou por serem repetidos.

\section{Avaliação do risco cardiovascular em comparação com a técnica do consultório}

\subsection{Normas de orientação clínica}

Em relação à AMPA, as Sociedades Europeia ${ }^{8}$ e Americana de Hipertensão ${ }^{14}$ encontram forte evidência em relação à melhor predição do risco $\mathrm{CV}$, identificando uma maioria de estudos que reportam esta conclusão. Existe um consenso generalizado, por parte dos autores de ambas as normas de orientação clínica, de que esta técnica de medição da PA deve, cada vez mais, ser parte integrante na rotina de avaliação do doente. Esta conclusão baseou-se na integração da evidência de vários parâmetros, entre os quais, a importância prognóstica adicional desta técnica.

Em relação à MAPA, a American Heart Association ${ }^{15}$ e a Sociedade Europeia de Hipertensão $0^{16}$ consideram haver vantagens desta técnica ao nível do prognóstico, justificando a posição apontando vários estudos com resultados concordantes (Quadro I). Estas sociedades recomendam a realização da MAPA em alguns subgrupos de hipertensos.

\subsection{Meta-análises}

Não foram seleccionadas meta-análises em relação à AMPA. Foram seleccionadas quatro meta-análises referentes à MAPA (Quadros II e III) com boa qualidade metodológica (nível de evidência 1). Todos os estudos englobados nas meta-análises seleccionadas são prospectivos. Para cada elemento do estudo, fazem a correlação entre os eventos CV e os valores de PA obtidos no início do estudo com a MAPA e a técnica do consultório. Os intervalos de estudo variam entre 1,9 e 11,1 anos.

As meta-análises de Hansen et $a l^{17} \mathrm{e}$ Conen et $a l^{18}$ (Quadro II) reportaram, para a população geral (hipertensos e normotensos), uma evidente superioridade prognóstica da PA medida pela MAPA em relação à obtida pela técnica do consultório. Por cada $10 \mathrm{mmHg}$ adicionais de pressão arterial sistólica (PAS), a medição da MAPA é entre 17 a $21 \%$ (p $\leq 0,001)$ mais fiável na predição do risco CV. Há ainda a relatar a perda da significância estatística da PA medida pela técnica do consultório no estudo de Conen. Os autores deste estudo encontraram, em relação à hipertensão mascarada e hipertensão da bata branca, um risco CV intermédio em relação à normalidade tensional e HTA.

Perante os resultados encontrados para a MAPA, e uma vez que na prática clínica é valorizada a relação entre as pressões arteriais diurnas e nocturnas, foi decidido realizar uma pesquisa suplementar incidindo no valor prognóstico diferencial das PA diurnas, nocturnas e suas relações. Neste desígnio, foram seleccionadas duas meta-análises ${ }^{19,20}$ englobando uma população total de 10926 indivíduos (Quadro III). Uma delas ${ }^{19}$ incidiu apenas em doentes hipertensos, fazendo análise separada para cada tipo de evento CV. A outra ${ }^{20}$ incidiu numa amostra aleatorizada da população com idade superior a 18 anos, englobando na mesma análise todos os eventos CV e apenas para a PAS. Apesar das diferenças metodológicas, os resultados foram concordantes. Em ambas, a PA nocturna foi a melhor preditora de eventos CV, comparativamente à PA diurna ou ao ratio diurno/nocturno. Esta superioridade da PA nocturna poderá advir, 


\begin{tabular}{|c|c|c|c|}
\hline Norma de orientação clínica (NOC) & Técnica & Ano & Conclusões \\
\hline $\begin{array}{l}\text { American Heart Association, American } \\
\text { Society of Hypertension \& Preventive CV } \\
\text { Nurses Association }{ }^{14}\end{array}$ & AMPA & 2008 & $\begin{array}{l}\text { A AMPA é um método mais fidedigno e melhor preditor do risco CV. } \\
\text { É tempo de tornar a AMPA rotina na avaliação dos doentes hipertensos, } \\
\text { particularmente em diabéticos, doentes coronários, insuficientes } \\
\text { renais, não aderentes à Tx ou com efeito da bata branca. }\end{array}$ \\
\hline European Society of Hypertension ${ }^{8}$ & AMPA & 2008 & $\begin{array}{l}\text { Atendendo à importância prognóstica da hipertensão mascarada e } \\
\text { aos benefícios prováveis no controlo e envolvimento no tratamento, } \\
\text { é recomendado o uso da AMPA em todos os hipertensos tratados. }\end{array}$ \\
\hline American Heart Association $^{15}$ & MAPA & 2005 & $\begin{array}{l}\text { Nível médio de PA correlaciona-se melhor com eventos CV que a } \\
\text { pressão do consultório. }\end{array}$ \\
\hline European Society of Hypertension ${ }^{16}$ & MAPA & 2007 & $\begin{array}{l}\text { Melhor correlação com lesão de órgãos-alvo e eventos CV. Maior } \\
\text { precisão na resposta da PA ao tratamento. }\end{array}$ \\
\hline
\end{tabular}

Legenda: CV, Cardiovasculares; NE, Nível de Evidência; Tx, Terapêutica.

QUADRO II. Meta-análises em relação à MAPA - Comparação desta técnica com a técnica do consultório em relação à capacidade de previsão de eventos CV.

\begin{tabular}{|c|c|c|c|c|c|c|c|c|c|}
\hline Estudo & $\begin{array}{c}\text { Tipo de } \\
\text { Comparação }\end{array}$ & $\begin{array}{c}\mathrm{N} \cdot{ }^{\circ} \mathrm{da} \\
\text { amostra }\end{array}$ & $\begin{array}{l}\text { População } \\
\text { estudada }\end{array}$ & $\begin{array}{l}\text { Follow- } \\
\text {-up } \\
\text { (anos) }\end{array}$ & $\begin{array}{l}\text { Eventos } \\
\text { CV }\end{array}$ & $\begin{array}{c}\text { Definição } \\
\text { eventos CV }\end{array}$ & $\begin{array}{c}\text { RR por cada } 1 \mathrm{~mm} \\
\text { MAPA } 24 \mathrm{~h}\end{array}$ & $\begin{array}{l}\mathrm{Hg} \text { (PAS/PAD) } \\
\text { PAMC }\end{array}$ & NE \\
\hline $\begin{array}{l}\text { IDACO }^{17} \\
2007\end{array}$ & $\begin{array}{l}\text { MAPA diurna } \\
\text { vs } \\
\text { PAMC }\end{array}$ & 7030 & $\begin{array}{l}\text { População } \\
\text { Geral }\end{array}$ & 9,5 & 863 & $\begin{array}{l}\text { Morte CV, } \\
\text { EAM, AVC, } \\
\text { ICC, RC }\end{array}$ & $\begin{array}{r}1,017 / 1.009 \\
p \leq 0,0001\end{array}$ & SSE & 1 \\
\hline $\begin{array}{l}\text { Conen }^{18} \\
2008\end{array}$ & $\begin{array}{l}\text { MAPA 24h } \\
\text { vs } \\
\text { PAMC }\end{array}$ & 19771 & $\begin{array}{l}\text { População } \\
\text { Geral }\end{array}$ & $\begin{array}{r}1,9- \\
-11,1\end{array}$ & 881 & $\begin{array}{l}\text { EAM, ICC, } \\
\text { RC, angina } \\
\text { instável, AVC, } \\
\text { morte CV }\end{array}$ & $\begin{array}{l}1,021 * /- \\
p \leq 0,001\end{array}$ & - & 1 \\
\hline
\end{tabular}

Legenda: RR, risco relativo; PAS, pressão arterial sistólica; PAD, pressão arterial diastólica; PAMC, pressão arterial medida no consultório; NE, nível de evidência; CV, Cardiovascular; EAM, Enfarte Agudo do Miocárdio; AVC, Acidente Vascular Cerebral; ICC, Insuficiência Cardíaca Congestiva; RC, Revascularização Cardíaca; SSE, sem significado estatístico ( $P>0,05)$.

*Foi calculado apenas a partir do ajustamento dos valores da PA obtidos pela MAPA com os obtidos no consultório, indicando valor preditivo independente da PA obtida pela MAPA.

por um lado, do seu carácter mais estável, e por outro, da sua menor susceptibilidade a factores externos (exercício físico, emoções). Considerando os dois estudos, o ratio foi o parâmetro que se relacionou em menor grau com os eventos $\mathrm{CV}$, não obtendo significância estatística no estudo IDACO II. ${ }^{20}$ No estudo de Fagard et al, ${ }^{19}$ por outro lado, só a PA nocturna manteve significância es- tatística quando esta e a PA diurna foram introduzidas no mesmo modelo estatístico.

\subsection{Estudos originais}

Foram seleccionados sete ECA em relação à AMPA e quatro estudos em relação à MAPA, dois ECA e dois observacionais, direccionados à análise prognóstica com- 


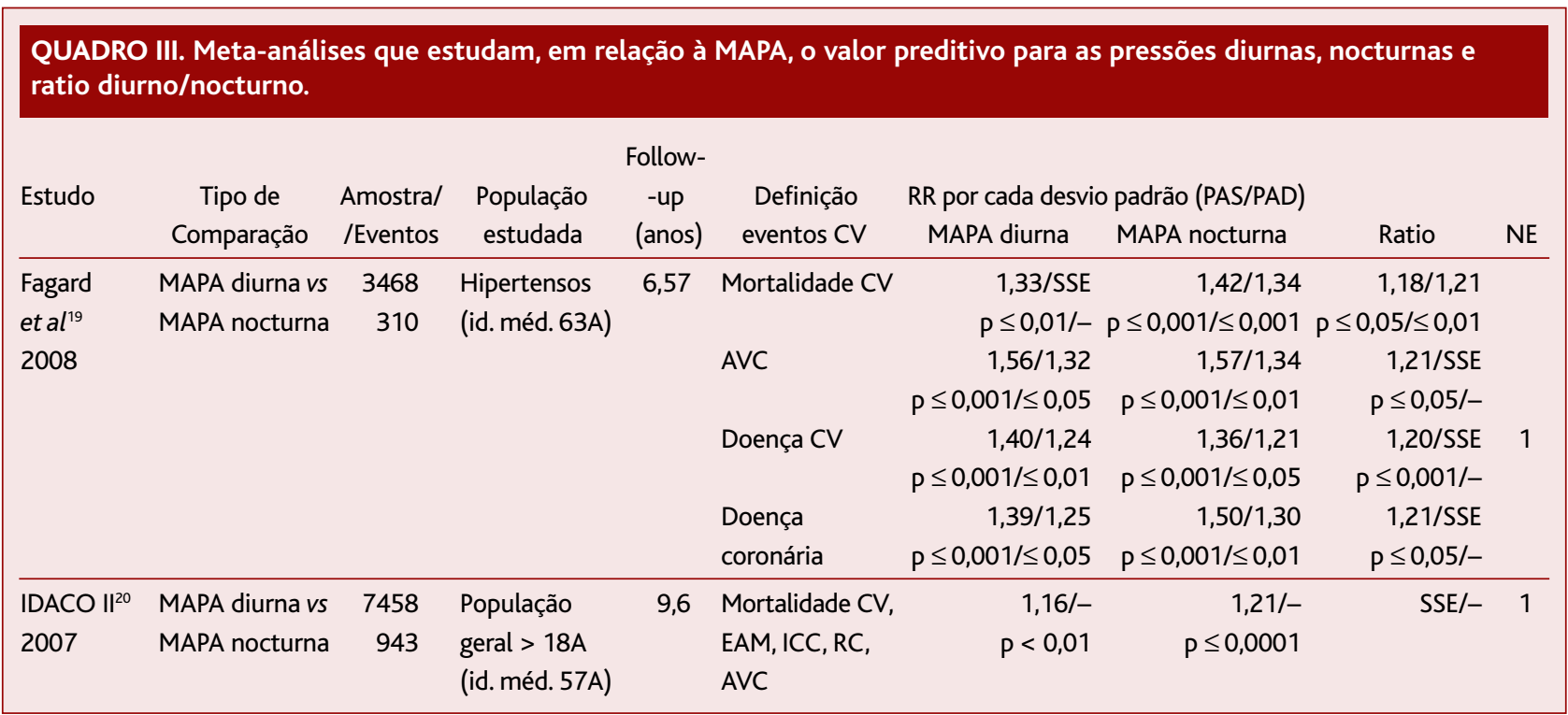

Legenda: RR, risco relativo; PAS, pressão arterial sistólica; PAD, pressão arterial diastólica; PAMC, pressão arterial medida no consultório; NE, nível de evidência; CV, Cardiovascular; EAM, Enfarte Agudo do Miocárdio; AVC, Acidente Vascular Cerebral; ICC, Insuficiência Cardíaca Congestiva; RC, Revascularização Cardíaca; SSE, sem significado estatístico $(p>0,05)$.

parativa com a técnica do consultório.

Os estudos compararam a relação entre os eventos CV e os valores de PA obtidos, no início do estudo, com a técnica do consultório e uma das técnicas do ambulatório (Quadro IV). Os intervalos de seguimento variaram entre os três e os 11 anos.

A maioria dos estudos incidiu em amostras de base populacional, dois deles focando-se apenas em hipertensos tratados. ${ }^{21-26} \mathrm{~A}$ técnica de medição da AMPA variou consideravelmente entre os estudos. Três deles (Belga ${ }^{23}$, Pamela $^{24} \mathrm{e} \mathrm{J-Health}{ }^{26}$ ) compararam as técnicas utilizando apenas uma ou duas medições para a AMPA, sendo que apenas o estudo mais recente (Finn-Home ${ }^{27}$ se aproximou da metodologia actualmente preconizada.

Alguns estudos incidiram em amostras relativamente pequenas (Belga, ${ }^{23}$ Didima $^{25}$ ) e, na sua maioria, obtiveram um número reduzido de end-points (Belga, ${ }^{23} \mathrm{~Pa}$ mela, ${ }^{24}$ Didima, ${ }^{25}$ e J-Health ${ }^{26}$, limitando o seu poder estatístico. Pelas limitações referidas, todos os estudos seleccionados foram classificados como nível de evidência 2, com excepção do estudo Finn-Home ${ }^{27}$ (nível de evidência 1), que incluiu uma amostra mais facilmente generalizável à população hipertensa, com um adequado tempo de seguimento, técnica de AMPA de acordo com as recomendações actuais e número de eventos adequado para obtenção de um razoável poder estatístico.

A maioria dos estudos identificam uma superioridade prognóstica da AMPA, incluindo os dois estudos em que esta técnica se baseou apenas em uma medição. A eliminação do efeito da bata branca parece ser o factor decisivo para a obtenção dos resultados. Em adição: vários estudos $^{21,23,25}$ não encontram relação estatisticamente significativa entre os valores de PAS da técnica do consultório e os eventos $\mathrm{CV}$; o estudo Ohasama ${ }^{22}$ apenas encontrou significância para valores superiores a 160/100mmHg (ou 140/90mmHg associado a factores de risco CV); o estudo J-Health ${ }^{26}$ apenas encontrou significância para PAS acima dos 160mmHg; o estudo Finn$\mathrm{Home}^{27}$ não encontrou significância para a mortalidade total, ao contrário do verificado para a AMPA.

$\mathrm{O}$ estudo Didima ${ }^{25}$ foi o único que obteve resultados discordantes, não encontrando valor prognóstico adicional para a AMPA. Porém, este estudo apresenta limitações em relação aos restantes. É de salientar a não ocorrência de relação estatisticamente significativa entre as PAS obtidas por ambas as técnicas e os eventos CV. O limitado poder estatístico deste estudo é sustentado ainda pela falha em estabelecer causalidade entre vários fac- 


\begin{tabular}{|c|c|c|c|c|c|c|c|c|c|c|c|}
\hline \multirow[t]{2}{*}{ Estudo } & \multirow[t]{2}{*}{ Tipo } & \multirow{2}{*}{$\begin{array}{c}\mathrm{N} .{ }^{\circ} \text { da } \\
\text { amostra }\end{array}$} & \multirow{2}{*}{$\begin{array}{c}\text { População } \\
\text { estudada }\end{array}$} & \multirow[b]{2}{*}{ Idade } & \multirow{2}{*}{$\begin{array}{l}\text { Follow- } \\
\text {-up } \\
\text { (anos) }\end{array}$} & \multirow{2}{*}{$\begin{array}{c}\text { Eventos } \\
\text { CV }\end{array}$} & \multirow{2}{*}{$\begin{array}{c}\text { Definição } \\
\text { eventos CV }\end{array}$} & \multirow{2}{*}{$\begin{array}{c}\text { Medições } \\
\text { AMPA }\end{array}$} & \multicolumn{2}{|c|}{ RR por cada $1 \mathrm{~mm} \mathrm{Hg \ddagger} \mathrm{(PAS/PAD)}$} & \multirow[b]{2}{*}{ NE } \\
\hline & & & & & & & & & AMPA & PAMC & \\
\hline $\begin{array}{l}\text { SHEAF }^{21} \\
2004\end{array}$ & ECA & 4932 & $\begin{array}{l}\text { Hipertensos } \\
\text { tratados }\end{array}$ & 70,0 & 3,2 & 324 & $\begin{array}{l}\text { Morte } \\
\text { CV,EAM, } \\
\text { AVC, TIA, } \\
\text { ICC, RC }\end{array}$ & $\begin{array}{r}4 \text { dias } \\
(3+3 / \text { dia })\end{array}$ & $\begin{array}{r}1,015 / 1,020 \\
p \leq 0,001 / \leq \\
0,001\end{array}$ & SSE & 2 \\
\hline $\begin{array}{l}\text { OHASAMA } 22 \\
2004\end{array}$ & ECA & 1702 & Geral & 60,6 & 10,6 & 141 & AVC & $\begin{array}{r}1 \text { mês } \\
\text { (1/dia) }\end{array}$ & $*$ & $*$ & 2 \\
\hline $\begin{array}{l}\text { BELGA }^{23} \\
2005\end{array}$ & ECA & 391 & Referenciada & 71,0 & 10,9 & 86 & $\begin{array}{l}\text { Morte CV, } \\
\text { EAM, AVC }\end{array}$ & Única & $\begin{array}{r}1,012 / 1,034 \\
P=0,01 / 0,001\end{array}$ & SSE & 2 \\
\hline $\begin{array}{l}\text { PAMELA }{ }^{24} \\
2005\end{array}$ & ECA & 2051 & Geral & 53,0 & 10,9 & 69 & Morte CV & $\begin{array}{r}1 \text { dia } \\
(1+1)\end{array}$ & $\begin{array}{r}1,046 / 1,055 \\
p \leq 0,001 / \leq \\
0,005\end{array}$ & $\begin{array}{r}1,030 / 1,044 \\
P \leq 0,001 / \leq 0,005\end{array}$ & 2 \\
\hline $\begin{array}{l}\text { DIDIMA }^{25} \\
2007\end{array}$ & ECA & 662 & Geral & 54,1 & 8,2 & 67 & $\begin{array}{l}\text { Morte CV, } \\
\text { EAM, AVC, } \\
\text { TIA, ICC, RC, } \\
\text { RAA, EAP }\end{array}$ & $\begin{array}{r}3 \text { dias } \\
(2+2 / \text { dia })\end{array}$ & SSE & $\begin{array}{r}\text { SSE } / 1,034 \\
P=0,01\end{array}$ & 2 \\
\hline $\begin{array}{l}\text { J-HEALTH }{ }^{26} \\
2008\end{array}$ & ECA & 4596 & $\begin{array}{l}\text { Hipertensos } \\
\text { tratados }\end{array}$ & 60,8 & 3,5 & 60 & $\begin{array}{l}\text { Morte CV, } \\
\text { EAM, AVC }\end{array}$ & $\begin{array}{r}1 \text { mês } \\
\text { (1/dia) }\end{array}$ & $*$ & $*$ & 2 \\
\hline $\begin{array}{l}\text { FINN-HOME }{ }^{27} \\
2010\end{array}$ & ECA & 2081 & Geral & 56,4 & 6,8 & 162 & $\begin{array}{l}\text { Morte CV, } \\
\text { EAM, AVC, } \\
\text { ICC, RC }\end{array}$ & $\begin{array}{r}7 \text { dias } \\
(2+2 / \text { dia })\end{array}$ & $\begin{array}{r}1,023 / 1,018 \\
p \leq 0,001 / \leq \\
0,001\end{array}$ & $P=\begin{array}{r}1,013 / 1,013 \\
0,001 / 0,002\end{array}$ & 1 \\
\hline
\end{tabular}

Legenda: RR, risco relativo; ECA, ensaio clínico aleatorizado; PAS, pressão arterial sistólica; PAD, pressão arterial diastólica; PAMC, pressão arterial medida no consultório; NE, nível de evidência; CV, cardiovascular; EAM, enfarte agudo do miocárdio; AVC, acidente vascular cerebral; AIT, acidente vascular cerebral transitório; ICC, insuficiência cardíaca congestiva; RC, revascularização cardíaca; RAA, rotura aneurima da aorta; EAP, edema agudo do pulmão; SSE, sem significado estatístico ( $p>0,05)$. ¥ Os RR dos estudos Belga e Pamela foram indicados para desvios padrão de PA, o cálculo para mmHg foi retirado do comentário editorial ao estudo Finn-Home, de Stergiou et al..$^{28}$ Para efeitos práticos, apenas se revela o valor dos RR estatisticamente significativos. Com excepção do estudo Pamela, ${ }^{24}$ todos os RR estão ajustados a factores como o tabagismo, ${ }^{21,23,25,27}$ idade, ${ }^{21,23,25,27}$ sexo, ${ }^{21,23,25,27}$ história de doença cardiovascular, ${ }^{21,25,27}$ diabetes mellitus, ${ }^{21,23,25,27}$ medicação anti-hipertensora, ${ }^{23,25,27}$ hipercolesterolémia, ${ }^{21,23,27}$ frequência cardíaca ${ }^{21}$ e índice de massa corporal. ${ }^{23}$

* Estimativas do risco não estão reportadas por $\mathrm{mmHg}$, mas por categorias de PA. Globalmente, mostram um aumento significativo do risco para eventos quanto maior for a PA medida pela AMPA, em grau mais significativo que a medida no consultório.

tores de risco CV, como história pessoal de doença CV e o tabagismo, com os eventos do estudo. Para além do reduzido número de eventos $\mathrm{CV}$, o estudo incluiu uma fracção significativa de jovens adultos saudáveis na amostra, diluindo significativamente o seu poder estatístico.

Para a MAPA (Quadro V), os ECA de Eguchi' ${ }^{28}$ (nível de evidência 2) e de Burr (Dublin) ${ }^{29}$ (nível de evidência 2) estão de acordo com os resultados prévios das metaanálises - maior correlação da MAPA para os eventos
CV futuros. O último tem a particularidade de ter avaliado a população idosa ( $>65$ anos), onde ainda havia um déficit de evidência. Nesta população, a prevalência de hipertensão mascarada pode ser mais elevada.

Já o estudo espanhol de Sierra ${ }^{30}$ (nível de evidência 2), centrado nos cuidados de saúde primários e de larga escala, analisou transversalmente a relação entre o perfil circadiano e os eventos CV e encontrou uma relação entre o perfil não dipper e lesão de órgãos-alvo e 


\begin{tabular}{|c|c|c|c|c|c|c|c|c|c|c|}
\hline \multirow[t]{2}{*}{ Estudo } & \multirow[t]{2}{*}{ Tipo } & \multirow{2}{*}{$\begin{array}{c}\mathrm{N} .{ }^{\circ} \mathrm{da} \\
\text { amostra }\end{array}$} & \multirow{2}{*}{$\begin{array}{l}\text { População } \\
\text { estudada }\end{array}$} & \multirow[b]{2}{*}{ Idade } & \multirow{2}{*}{$\begin{array}{c}\text { Follow-up } \\
\text { (anos) }\end{array}$} & \multirow{2}{*}{$\begin{array}{c}\text { Eventos } \\
\text { CV }\end{array}$} & \multirow{2}{*}{$\begin{array}{l}\text { Definição } \\
\text { eventos CV }\end{array}$} & \multicolumn{2}{|c|}{ RR (PAS/PAD) } & \multirow[b]{2}{*}{ NE } \\
\hline & & & & & & & & MAPA & PAMC & \\
\hline $\begin{array}{l}\text { Eguchi et } \\
a^{28}\end{array}$ & ECA & 1268 & Hipertensos & 70,4 & 4,2 & 100 & $\begin{array}{l}\text { AVC, EAM fatal } \\
\text { ou não-fatal, } \\
\text { morte súbita } \\
\text { cardíaca }\end{array}$ & 1,044/- & SSE* & 1 \\
\hline $\begin{array}{l}\text { DUBLIN }{ }^{29} \\
2008\end{array}$ & ECA & 1144 & Idosos $(>65 \mathrm{~A})$ & 73,1 & 6,7 & 246 & Morte CV & $1,015 / 1,008^{*}$ & SSE* & 2 \\
\hline $\begin{array}{l}\text { Sierra et } \\
a l^{30} \\
2009\end{array}$ & $\begin{array}{l}\text { Observa- } \\
\text { cional }\end{array}$ & 42947 & Hipertensos & 53,2 & - & - & & $\begin{array}{l}\text { Não dipper: associa } \\
\text { e complicaçõe }\end{array}$ & $\begin{array}{l}\text { do a HVE } \\
\text { s CV. }\end{array}$ & \\
\hline $\begin{array}{l}\text { Mesquita } \\
\text { Bastos et } \\
a^{31} \\
2010\end{array}$ & $\begin{array}{l}\text { Observa- } \\
\text { cional }\end{array}$ & 1200 & Hipertensos & 51,0 & 8,2 & 152 & $\begin{array}{l}\text { Eventos ou } \\
\text { morte por ICC, } \\
\text { ECV, EAM, } \\
\text { angina, RC }\end{array}$ & $\begin{array}{r}1,58 / 1,23(24 \mathrm{~h})^{* *} \\
1,48 / \mathrm{SSE}(\text { dia) } \\
1,66 / 1,40 \text { (noite) }^{* *} \\
1,28 / 1,29 \text { (ratio) }^{* *}\end{array}$ & $1,02 / \mathrm{SSE}^{* *}$ & 2 \\
\hline
\end{tabular}

Legenda: PAS, pressão arterial sistólica; PAD, pressão arterial diastólica; ECA, ensaio clínico aleatorizado; NE, nível de evidência; PAMC, pressão arterial medida no consultório; SSE, sem significado estatístico ( $p$ > 0,05); CV, Cardiovascular; HVE, hipertrofia ventricular esquerda; ICC, Insuficiência Cardíaca Congestiva; EVC, Eventos Cerebrovasculares; RC, Revascularização Cardíaca.

* RR apresentados por cada aumento de $1 \mathrm{mmHg}$;

** RR apresentados por cada aumento de um desvio padrão de PA.

complicações CV. Contudo, não explorou se esta relação era superior às obtidas pelos valores diurnos ou nocturnos da PA.

Num coorte de 1200 indivíduos portugueses referenciados para a realização de MAPA entre 1991 e 1998, o estudo retrospectivo de Mesquita Bastos ${ }^{31}$ (nível de evidência 2) identificou, 15 anos após a realização da MAPA, uma franca superioridade prognóstica da técnica. A PA medida no consultório revelou-se um fraco preditor dos eventos CV no geral (RR 1,02) e sem significância estatística para eventos coronários. Em concordância com as meta-análises referidas anteriormente, a PA nocturna foi melhor preditora dos eventos, quando comparada com a PA diurna ou o ratio diurno/nocturno, sendo o valor deste novamente questionado face à sua baixa reprodutibilidade.

\section{Controlo no seguimento do hipertenso} comparativamente à técnica do consultório

\subsection{Normas de Orientação Clínica}

Em relação à AMPA, a Sociedade Europeia da Hipertensão ${ }^{8}$ identifica, através da análise de diversos estu- dos, benefícios prováveis no controlo da PA e envolvimento do utente no seu tratamento.

\subsection{Meta-análises e Revisões Sistemáticas}

Para a AMPA, foram seleccionadas duas revisões sistemáticas ${ }^{32,33}$ e quatro meta-análises ${ }^{34-37}$ (Quadro VI), todas com um protocolo bem estruturado, detalhado e explícito (nível de evidência 1).

No estudo de Verberk, ${ }^{32}$ os estudos englobados realizam uma comparação entre os valores de PA obtidos com a AMPA e com a técnica do consultório, para cada um dos indivíduos seleccionados. Os resultados mostram que, quando se mede a PA com as duas técnicas, num determinado período, a AMPA associa-se a valores de PA significativamente mais baixos (Quadro VI), sendo a probabilidade de diferença maior nos hipertensos não tratados. Na origem desta diferença está a eliminação do efeito da bata branca. Na revisão da Cochrane, ${ }^{33}$ a AMPA foi das poucas intervenções que provou ter eficácia na redução da PA.

As meta-análises de Capuccio ${ }^{34}$ (nível de evidência 1), Bray $^{35}$ (nível de evidência 1) e Agarwal ${ }^{36}$ (nível de evidên- 


\begin{tabular}{|c|c|c|c|c|c|c|}
\hline \multicolumn{7}{|c|}{$\begin{array}{l}\text { QUADRO VI. Revisões sistemáticas }{ }^{30,31} \text { e meta-análises }{ }^{31,33,35} \text { referentes ao estudo do controlo da HTA com a AMPA }^{30,31,32,3} \\
\text { e a MAPA. }^{34}\end{array}$} \\
\hline Estudo & Técnica & $\begin{array}{l}\text { Tipo de } \\
\text { estudo }\end{array}$ & $\begin{array}{c}\text { N. } .^{\circ} \text { da } \\
\text { amostra }\end{array}$ & $\begin{array}{l}\text { População } \\
\text { estudada }\end{array}$ & Resultados & NE \\
\hline $\begin{array}{l}\text { Verberk et al } \\
2005\end{array}$ & AMPA & $\begin{array}{l}\text { Revisão } \\
\text { Sistemática }\end{array}$ & 15907 & $\begin{array}{l}\text { Hipertensos } \\
\text { tratados e não } \\
\text { tratados }\end{array}$ & $\begin{array}{l}\text { Valores PAS/PAD mais baixos }(5,3-6,9 / 3,1-4,9 \mathrm{~mm} \\
\mathrm{Hg}, \mathrm{p}<0,001) \text {. } \\
\text { Resposta terapêutica: descida menos pronunciada. }\end{array}$ & 1 \\
\hline $\begin{array}{l}\text { Fahey et al }{ }^{33} \\
\text { (Cochrane) } \\
\text { 2004 }\end{array}$ & AMPA & $\begin{array}{l}\text { Revisão } \\
\text { sistemática }\end{array}$ & $\begin{array}{l}\text { Não } \\
\text { reportado }\end{array}$ & Hipertensos & $\begin{array}{l}\text { Diminuição PAM 2,0mmHg ( } 95 \% \text { IC -2,7 a -1,4); Taxa } \\
\text { de controlo PA: tendência melhor controlo (mas sem } \\
\text { atingir significado estatístico). }\end{array}$ & 1 \\
\hline $\begin{array}{l}\text { Cappuccio et al }{ }^{34} \\
2004\end{array}$ & AMPA & Meta-análise & 2714 & Hipertensos & $\begin{array}{l}\text { Diminuição PAS 4,25mmHg ( } 95 \% \text { IC: }-6,95 \text { a -1,55); } \\
\text { Diminuição PAD 2,37mmHg (95\% IC: }-3,49 \text { a -2,37); } \\
\text { Taxa de controlo PA: RR 1,11 (95\% IC 1,00 a 1,24) - } \\
\text { valores PA mais baixos 2,2/1,9 mm Hg. }\end{array}$ & 1 \\
\hline $\begin{array}{l}\text { Bray et al } \\
2010\end{array}$ & AMPA & Meta-análise & 7119 & $\begin{array}{l}\text { Hipertensos } \\
\text { ou PA elevada }\end{array}$ & $\begin{array}{l}\text { Diminuição PAS 3,62mmHg (95\% IC -5,61 a -2,03); } \\
\text { Diminuição PAD 1,45mmHg (95\% IC -1,95 a -0,94); } \\
\text { Taxa de controlo PA: RR } 1.09 \text { (95\% IC 1,02 a 1,16). }\end{array}$ & 1 \\
\hline $\begin{array}{l}\text { Agarwal et } a l^{36} \\
2011\end{array}$ & AMPA & Meta-análise & 9446 & Hipertensos & $\begin{array}{l}\text { Diminuição PAS 2,63mmHg (95\% IC -4,24 a -1,02); } \\
\text { Diminuição PAD 1,68mmHg (95\% IC -2,58 a -0,79); } \\
\text { Taxa de controlo PA: RR 1,11 (95\% IC 0,97 a 1,26); } \\
\text { Diminuição medicação: RR 2,02 (95\% IC 1,32 a 3,11). }\end{array}$ & 1 \\
\hline $\begin{array}{l}\text { Ishikawa et al }{ }^{37} \\
2008\end{array}$ & AMPA & Meta-análise & 6742 & $\begin{array}{l}\text { Hipertensos } \\
\text { tratados }\end{array}$ & $\begin{array}{l}\text { Resposta terapêutica: descida menos pronunciada em } \\
20 \%(-15,2 /-10,3 \mathrm{mmHg}, \mathrm{p}<0,001)\end{array}$ & 1 \\
\hline $\begin{array}{l}\text { Mancia }^{38} \\
2004\end{array}$ & MAPA & Meta-análise & 6000 & Hipertensos & $\begin{array}{l}\text { Resposta terapêutica descida da PA menos } \\
\text { significativa em } 36 \% \text {. Redução da PA nocturna menor } \\
\text { que diurna. }\end{array}$ & 1 \\
\hline
\end{tabular}

Legenda: PAS, pressão arterial sistólica; PAD, pressão arterial diastólica; PAM, pressão arterial média; NE, nível de evidência.

cia 1) incluíram apenas ensaios clínicos que aleatorizaram os indíviduos pela AMPA (intervenção) e pela técnica do consultório (controlo).Verificou-se que a utilização da AMPA, por si só, ou associada a uma estratégia de resposta terapêutica, se traduziu em valores significativamente mais baixos de PAS (descida até 4,25mmHg) e PAD (descida até $2,37 \mathrm{mmHg}$ ) no consultório. As descidas nos valores de PA originaram aumentos das taxas de controlo tensional entre $9 \%$ a $11 \%$ nos estudos, estatisticamente significativos excepto na meta-análise de Agarwal $^{36}$ (tornando-se, no entanto, significativos quando excluiu os estudos de menor qualidade metodológica).

A associação de uma estratégia de resposta terapêutica aos valores obtidos pela AMPA é um factor que aumenta a probabilidade de melhor controlo. ${ }^{34,35,36}$ Quando incluiu apenas os estudos que cumpriam este crité- rio, os resultados de Agarwal ${ }^{36}$ adquiriram significância estatística. Outros factores que podem estar na origem dos valores mais baixos da AMPA incluem: (1) diminuição da inércia clínica, ${ }^{35}$ (2) capacitação dos doentes em relação à gestão da HTA e aumento do cumprimento terapêutico, ${ }^{14,35}$ (3) o fenómeno da "habituação" a medições repetidas da PA..$^{34,35}$

Quando se avalia o fenómeno específico da resposta terapêutica, ${ }^{32,37}$ ou seja, a repercussão na PA após a introdução de um novo fármaco, como na meta-análise de Ishikawa ${ }^{37}$ (nível de evidência 1), uma situação diferente ocorre: ambas as técnicas identificam a esperada descida da PA, mas a AMPA revela-a em grau menos acentuado. Existe, portanto, uma sobre-estimação dos efeitos da instituição terapêutica com o método do consultório. Esta situação, em aparente contradição 
com a anterior, pode ter várias justificações segundo os autores. Em primeiro lugar, os valores iniciais pelo método AMPA não são tão elevados devido à ausência do efeito da bata branca. Por outro lado, parece ser descrita uma tendência natural dos clínicos no arredondamento favorável do valor medido da PA, na expectativa inconsciente da eficácia do tratamento imposto. Por último, a casualidade da medição do consultório pode aproximar-se mais do pico do período da actuação do anti-hipertensor instituído.

Já no que diz respeito à MAPA (Quadro VI), a metaanálise seleccionada avaliou apenas a resposta terapêutica. Tal como para a AMPA, a meta-análise ampla de Mancia ${ }^{38}$ encontrou uma resposta terapêutica menos acentuada, em grau ainda mais significativo que a relatada em média para a AMPA (36\%).

\subsection{Estudos originais}

Foram seleccionados dois ECA e um ensaio clínico aleatorizado e controlado (ECAC) relativos à AMPA e quatro estudos, três ECA e um ECAC, relativos à MAPA.

Os estudos originais seleccionados para a AMPA ${ }^{39,40,41}$ (Quadro VII), um dos quais em contexto nacional, mostram resultados semelhantes aos apresentados nas meta-análises - sustentam a observação de um melhor controlo da PA com a utilização da AMPA.

No estudo português ${ }^{39}$ (nível de evidência 1), para cada doente hipertenso as taxas de controlo tensional foram de $37,2 \%$, quando aferida pela técnica do con- sultório, e de 48,6\%, quando aferida pela AMPA. Face a estes resultados, e à expressão significativa da hipertensão da bata branca $(19,4 \%)$ e da hipertensão mascarada (8\%) naquela população portuguesa, Maldonado e colegas concluem existir uma superior adequação da AMPA à monitorização da PA, especialmente em indivíduos com maior risco CV, nos quais a informação obtida pela medição do consultório se revelou insuficiente. Os resultados do estudo português estão de acordo com as recomendações europeias no sentido da exclusão dos valores do primeiro dia de registo da AMPA por se verificarem valores significativamente mais elevados (sujeitos ainda a parte do efeito da bata branca que se perde com a habituação à técnica).

Já o estudo J-Home ${ }^{40}$ (nível de evidência 2) utilizou um amplo intervalo (2 semanas) para a obtenção do valor da AMPA, o que condicionou a obtenção dos resultados. Durante este intervalo, os clínicos tiveram total liberdade para agendar consultas de seguimento bem como para alterar a terapêutica. Este viés condicionou uma maior agressividade terapêutica registada no grupo avaliado com a AMPA.

Recentemente, o ECAC de McManus ${ }^{41}$ (nível de evidência 1) teve a particularidade de comparar a AMPA, associada a uma estratégia de auto-gestão da medicação por parte dos doentes e telemonitorização, com a estratégia habitual. Aos doentes foi fornecido, pelo médico de família, um algorítmo individualizado para ajuste simples (até dois passos) da medicação ao

\begin{tabular}{|c|c|c|c|c|c|c|c|c|}
\hline Estudo & Tipo & $\begin{array}{l}\mathrm{N} \cdot{ }^{\circ} \mathrm{da} \\
\text { amostra }\end{array}$ & $\begin{array}{l}\text { População } \\
\text { estudada }\end{array}$ & Idade & $\begin{array}{l}\text { Técnica } \\
\text { AMPA }\end{array}$ & $\begin{array}{l}\text { Follow- } \\
\text {-up }\end{array}$ & Resultados & $\mathrm{NE}$ \\
\hline $\begin{array}{l}\text { Estudo } \\
\text { AMPA }^{39} \\
2009\end{array}$ & ECA & 685 & $\begin{array}{l}\text { Hipertensos } \\
\text { tratados }\end{array}$ & 54,2 & $\begin{array}{r}5 \text { dias } \\
(3+3 / \text { dia })\end{array}$ & $5 d$ & $\begin{array}{l}\text { Taxa controlo tensional superior em } 30,6 \%(p<0,001) \\
\text { inerente à eliminação do efeito bata branca. } \\
\text { Discordância em } 27,4 \% \text { : HBB } 19,4 \% \text { e HM } 8 \% \text {. } \\
\text { AMPA permite melhor caracterização do perfil tensional. }\end{array}$ & 1 \\
\hline $\begin{array}{l}\text { J-Home } \\
2008\end{array}$ & ECA & 2363 & $\begin{array}{l}\text { Hipertensos } \\
\text { tratados }\end{array}$ & 66,3 & $\begin{array}{l}2 \text { semanas } \\
(1+1 / \text { dia })\end{array}$ & $2 \mathrm{~s}$ & Taxa controlo tensional superior em $23 \%(p<0,01)$ & 2 \\
\hline $\begin{array}{l}\text { TASMINH2 } 2^{41} \\
2010\end{array}$ & ECA & 527 & $\begin{array}{l}\text { Hipertensos } \\
\text { tratados }\end{array}$ & 66,4 & $\begin{array}{r}7 \text { dias } \\
(2+2 / \text { dia }) \\
1 x / \text { mês }\end{array}$ & $12 \mathrm{~m}$ & $\begin{array}{l}\text { Aos 6M: redução PAS } 3,7 \text { mmHg }(95 \% \text { IC 0,8-6,6) } \\
\text { Aos 12M: redução PAS } 5,4 \text { mmHg }(95 \% \text { IC } 2,4-8,5)\end{array}$ & 1 \\
\hline
\end{tabular}

Legenda: ECA, ensaio clínico aleatorizado; NE, nível de evidência; HBB, Hipertensão da Bata Branca; HM, Hipertensão Mascarada. 


\begin{tabular}{|c|c|c|c|c|c|c|}
\hline Estudo & Tipo & $\begin{array}{c}\mathrm{N} .{ }^{\circ} \text { da } \\
\text { amostra }\end{array}$ & $\begin{array}{l}\text { População } \\
\text { estudada }\end{array}$ & Idade & Resultados & NE \\
\hline $\begin{array}{l}\text { Manning }{ }^{42} \\
2006\end{array}$ & ECA & 514 & $\begin{array}{l}\text { Hipertensos } \\
\text { tratados }\end{array}$ & $50-75$ & Taxa controlo tensional superior em $57 \%, p<0,001$ & 2 \\
\hline $\begin{array}{l}\text { Banegas }^{43} \\
2007\end{array}$ & ECA & 12897 & $\begin{array}{l}\text { Hipertensos } \\
\text { tratados }\end{array}$ & 61,9 & $\begin{array}{l}\text { Taxa controlo tensional superior em } 28 \%(p<0,001) \\
\text { Identificação de HBB em } 33,4 \% \text { e HM em } 5,4 \% \text {. }\end{array}$ & 1 \\
\hline $\begin{array}{l}\text { Conen }^{44} \\
2009\end{array}$ & ECA & 165 & $\begin{array}{l}\text { Hipertensos } \\
\text { tratados }\end{array}$ & 56,0 & Taxa controlo tensional superior em $46,6 \%, p=0,04$ & 2 \\
\hline $\begin{array}{l}\text { MARTE }{ }^{45} \\
2010\end{array}$ & ECA & 2059 & $\begin{array}{l}\text { Hipertensos } \\
\text { tratados }\end{array}$ & 61,1 & $\begin{array}{l}\text { Concordância pobre entre PAMC e MAPA: controlados com PAMC }-66 \% \\
\text { probabilidade de não o estar com MAPA; não controlados com PAMC }-70 \% \\
\text { probabilidade de o estar com MAPA ( }<<0,001)\end{array}$ & 1 \\
\hline
\end{tabular}

Legenda: NE, nível de evidência; ECA: ensaio clínico aleatorizado; HBB, hipertensão da bata branca; HM, hipertensão mascarada; PAMC, pressão arterial medida no consultório.

resultado da AMPA (realizada uma vez por mês, durante uma semana). A estratégia resultou numa redução significativa da PAS (o parâmetro avaliado) equivalente a uma estimativa de redução do risco de AVC em $20 \%$ e de doença coronária em $10 \%$.

Tal como o observado para a AMPA, a generalidade dos estudos originais referentes à utilização da MAPA reportam taxas mais elevadas de controlo tensional, entre $24 \%$ a $57 \%$ (Quadro VIII). ${ }^{42-45}$

Os estudos foram aplicados no contexto do seguimento de hipertensos tratados em cuidados de saúde primários, na Suiça, ${ }^{42}$ Espanha (de larga escala), ${ }^{43}$ Reino Unido ${ }^{44}$ e Itália. ${ }^{45}$ Nestes estudos, para cada hipertenso, a MAPA revelou, em média, valores mais baixos do que a PA medida pela técnica do consultório e, por inerência, maiores taxas de controlo. No estudo italiano, ao contrário dos anteriores, a fraca concordância entre as pressões arteriais medidas no consultório e pela MAPA foi originada sobretudo pelo efeito da hipertensão mascarada e não pelo efeito da bata branca. No entanto, na maioria dos estudos realizados em contexto de cuidados de saúde primários, um dos quais realizado na população portuguesa, ${ }^{39}$ a hipertensão da bata branca tem maior peso. Os estudos suiço $\mathrm{O}^{42} \mathrm{e}$ britânico ${ }^{44}$ foram considerados com nível de evidência 2 pelo inferior poder estatístico inerente a amostras mais reduzidas, e por falhas na caracterização inicial da população. Os dois restantes estudos foram considerados bons sob o ponto de vista metodológico (nível de evidência 1).

Assim, verificou-se, para ambas as técnicas, uma discordância entre os valores de PA obtidos com estas e no consultório, com repercussões ao nível do controlo da HTA.

\section{Relação custo-benefício comparativamente à utilização isolada da técnica convencional do consultório}

Ao permitirem eliminar o efeito da bata branca e, consequentemente, eliminarem o eventual uso de medicação numa fracção considerável de utentes, foi colocada como hipótese as técnicas do ambulatório apresentarem um custo-benefício favorável. Sobre este aspecto, foram seleccionados cinco estudos, dois observacionais e três ensaios clínicos (Quadro IX).

$\mathrm{O}$ estudo transversal de Fukunaga et a $\boldsymbol{l}^{46}$ (nível de evidência 1) estimou os custos para o sistema de saúde japonês caso a AMPA fosse implementada a todos os indivíduos rastreados como hipertensos pelo método do consultório. Estes dados têm em conta a cobertura total dos custos com os aparelhos de AMPA por parte dos utentes. Considerando uma incidência anual média de $7,4 \%$ de hipertensos do consultório na população japonesa, identificou uma redução média de 1,56 milhões de dólares por 1000 indivíduos em cinco anos. Esta redução de custos é proporcional ao fenómeno da 


\begin{tabular}{|c|c|c|c|c|c|c|c|c|}
\hline Estudo & Técnica & $\begin{array}{l}\text { Tipo de } \\
\text { estudo }\end{array}$ & Amostra & $\begin{array}{r}\text { HBB } \\
(\%)\end{array}$ & $\begin{array}{l}\text { Follow- } \\
\text {-up } \\
\text { (anos) }\end{array}$ & $\begin{array}{c}\text { Cut-off } \\
\text { diagnóstico }\end{array}$ & Resultados & NE \\
\hline $\begin{array}{l}\text { Fukunaga }{ }^{46} \\
2008\end{array}$ & AMPA & $\begin{array}{l}\text { Observa- } \\
\text { cional }\end{array}$ & $\begin{array}{l}\text { População } \\
\text { japonesa }\end{array}$ & 16,5 & 5 & $<135 / 85 \mathrm{mmHg}$ & $\begin{array}{l}\text { Redução de custos mínima de } 674 \text { mil dólares } \\
\text { (HBB 8,2\%) e máxima de } 2.51 \text { milhões de dólares } \\
\text { (HBB 24,7\%) por cada } 1000 \text { indivíduos - redução } \\
\text { entre 6,2 a 23\% nos gastos com a HTA, } \\
\text { proporcional ao fenómeno da HBB. }\end{array}$ & 1 \\
\hline $\begin{array}{l}\text { Krakoff }^{47} \\
2006\end{array}$ & MAPA & $\begin{array}{l}\text { Observa- } \\
\text { cional }\end{array}$ & $\begin{array}{l}\text { População } \\
\text { americana }\end{array}$ & 20 & 5 & $\begin{array}{r}<135 / 85 \mathrm{mmHg} \\
\text { (diurna) } \\
<130 / 80 \mathrm{mmHg} \\
(24 \mathrm{~h})\end{array}$ & $\begin{array}{l}\text { Estratégia com MAPA reduz número de } \\
\text { tratamentos/ano entre } 10 \text { a } 23 \% \text {, proporcional à } \\
\text { incidência de HTA. Redução de custos média de } \\
334 \text { dólares por utente. }\end{array}$ & 1 \\
\hline $\begin{array}{l}\text { Ewald } 48 \\
2002\end{array}$ & MAPA & ECA & 62 & 26 & 7 & $\begin{array}{r}<130 / 85 \mathrm{mmHg} \\
(\text { diurna M) } \\
<135 / 86 \mathrm{mmHg} \\
(\text { diurna H) }\end{array}$ & $\begin{array}{l}\text { Ao fim de } 3 \text { anos, qualquer estratégia de utilização } \\
\text { da MAPA estudada (anual, bienal ou trienal) } \\
\text { começa a compensar os custos iniciais, por redução } \\
\text { do número de tratamentos/ano. Redução total } \\
\text { de custos entre } 8 \text { (anual) a 13\% (trienal). }\end{array}$ & 1 \\
\hline $\begin{array}{l}\text { Lorgelly }{ }^{49} \\
2003\end{array}$ & MAPA & ECA & 374 & 31 & 2 & $\begin{array}{r}<140 / 85 \mathrm{mmHg} \\
(24 \mathrm{~h})\end{array}$ & $\begin{array}{l}\text { MAPA reduz número de tratamentos/ano mas } \\
\text { insuficiente para ter relação custo-benefício } \\
\text { favorável no intervalo do estudo. }\end{array}$ & 2 \\
\hline $\begin{array}{l}\text { Pessanha }^{50} \\
2008\end{array}$ & MAPA & ECA & 336 & 38,7 & 1,33 & $\begin{array}{r}<135 / 85 \mathrm{mmHg} \\
\text { (diurna) }\end{array}$ & $\begin{array}{l}\text { Uso generalizado da MAPA nos cuidados de } \\
\text { saúde primários permite reduzir significativamente } \\
\text { os gastos em saúde e os riscos de iatrogenia } \\
\text { medicamentosa. }\end{array}$ & 2 \\
\hline
\end{tabular}

Legenda: $H B B$, hipertensão da bata branca; $N E$, nível de evidência; $m$, média; $M$, sexo feminino; $H$, sexo masculino; $E C A$, ensaio clínico aleatorizado.

hipertensão da bata branca.

Apesar de gozar da fama de uma técnica dispendiosa, a verdade é que vários estudos têm demonstrado, também para a MAPA, uma favorável relação de custobenefício, ${ }^{47-50}$ sobretudo a longo prazo.

O estudo transversal de Krakoff ${ }^{47}$ (nível de evidência 1) estimou uma poupança de $3 \%$ a $14 \%$ nos custos associados à HTA e uma redução de $10 \%$ a $23 \%$ nos dias de tratamento, para um período de cinco anos, caso a MAPA fosse incorporada no algoritmo diagnóstico de todos os novos hipertensos americanos. Esta análise teve em conta a prevalência de $20 \%$ de hipertensão da bata branca no rastreio inicial com a técnica do consultório, a estimativa de incidência anual de HTA, os custos associados à MAPA e os custos associados ao seguimento e tratamento dos hipertensos.

Os três restantes ensaios clínicos focam-se na reali- dade dos cuidados de saúde primários da Austrália, ${ }^{48}$ Reino Unido ${ }^{49} \mathrm{e}$ Portugal. ${ }^{50}$

O estudo australiano ${ }^{48}$ (nível de evidência 1) seguiu 62 doentes durante sete anos e realizou uma análise do custo-benefício para vários modelos de utilização da MAPA - anual, bienal e trienal. A hipertensão da bata branca foi identificada em $26 \%$ dos utentes. Ao terceiro ano, todas as estratégias de monitorização compensam os gastos iniciais e, a partir daí, têm uma relação custo-benefício muito favorável.

Por sua vez, o estudo de Lorgelly et a ${ }^{49}$ (nível de evidência 2) realizou uma análise ao custo-benefício da MAPA anual a 374 hipertensos seguidos durante dois anos numa unidade de cuidados de saúde primários do Reino Unido. Identificou 31\% de doentes com efeito da bata branca que, sem a utilização da MAPA, teriam potencial para serem submetidos a intervenção e consul- 
tas de seguimento desnecessárias. Em situação oposta, identificou $6 \%$ de doentes nos quais os valores de PA do consultório mas não da MAPA estavam bem controlados. Este foi o único estudo que teve em atenção este fenómeno inverso. Considerando as duas situações, e os gastos inerentes à utilização da MAPA (13790 libras/ano), a utilização desta no seguimento dos hipertensos atenuaria os gastos. No entanto, no que diz respeito à relação custo-benefício, o intervalo de follow- up de dois anos foi insuficiente para se verificar um efeito positivo (-3612 libras/ano).

Em Portugal, o estudo de Pessanha et a ${ }^{5^{0}}$ (nível de evidência 2) identificou uma prevalência de hipertensão da bata branca de $38,7 \%$, calculada de uma amostra de 336 hipertensos do consultório seguidos durante 16 meses. Ao contrário dos resultados do estudo britânico, foi verificada uma relação custo-benefício favorável com a utilização da MAPA já ao fim dos dois anos: redução de $23 \%$ dos custos associados à terapêutica anti-hipertensora em procedimentos médicos de diagnóstico e seguimento. Para estes resultados foram considerados os custos de aquisição dos aparelhos MAPA por parte da unidade, formação dos profissionais de saúde e consultas médicas de seguimento, incluindo tratamento e exames complementares de diagnóstico.

\section{CONCLUSÕES}

Foi realizada uma análise à implementação de cada uma das técnicas de medição da PA do ambulatório (MAPA e AMPA) comparativamente ao uso isolado da técnica do consultório. A comparação incidiu em três domínios: identificação do risco CV, avaliação no seguimento do doente hipertenso e relação custo-benefício. Estes foram considerados os domínios essenciais para a avaliação destas técnicas como parte integrante do estudo e avaliação do doente hipertenso. Foi decidido englobar num mesmo estudo a MAPA e a AMPA pela raridade de estudos que as incluem num mesmo modelo de investigação. Procurou-se, assim, contrariar um certo distanciamento científico prejudicial entre as duas.

Apesar de terem sido considerados fundamentais os princípios referidos, os autores reconhecem uma maior dificuldade metodológica subjacente a um estudo de revisão baseada na evidência tão amplo. Porém, é total convicção dos autores terem sido cumpridos todos os requisitos científicos e que as conclusões do estudo reflectem a melhor evidência actual.

Em relação à identificação do risco CV, existe evidência para a utilização das técnicas do ambulatório com o objectivo de obter avaliações diagnóstica e prognóstica mais fidedignas - força de recomendação A para a MAPA e força de recomendação B para a AMPA. Especificamente em relação à MAPA, foi identificada evidência de uma superioridade prognóstica da PA nocturna em relação à PA diurna. Não existe evidência para sobrevalorizar, em termos prognósticos, a relação entre a PA diurna e nocturna (status dipping) em relação à PA nocturna por si mesma - força de recomendação A.

No que diz respeito à avaliação no seguimento do doente hipertenso, existe evidência sólida para a utilização de ambas as técnicas de medição do ambulatório (MAPA e AMPA) com o intuito de obter um controlo mais adequado da PA, ou seja, que uma percentagem maior de hipertensos atinjam os valores-alvo pretendidos - força de recomendação A.

Por último, existe evidência para a aplicação das técnicas do ambulatório a todos os novos hipertensos do consultório e durante o seguimento destes com o propósito de reduzir os custos associados à HTA - força de recomendação $\mathrm{B}$.

O Quadro X resume as principais conclusões relativamente aos aspectos estudados para cada uma das técnicas do ambulatório.

Assim, a medição do consultório, não obstante continuar a ser fundamental no processo de avaliação da $\mathrm{PA}$, pode limitar a prestação dos melhores cuidados ao doente hipertenso, quando usada isoladamente. Os autores concluem existir uma evidência premente para a integração da medição da PA no ambulatório no processo de avaliação diagnóstica e seguimento do doente hipertenso.

Das duas técnicas, existem áreas de evidência convincente e outras onde ainda existe alguma incerteza nas quais mais estudos são necessários. A AMPA requer mais estudos de qualidade que incidam sobre o intervalo mais adequado para as medições e a exclusão ou não do primeiro dia de registos. A falta de uniformização internacional destes aspectos estruturantes tem-se reflectido nos estudos e prejudicado a sua investigação.

Serão igualmente necessários no futuro estudos de qualidade que incluam AMPA e MAPA no mesmo pro- 


\begin{tabular}{|c|c|c|}
\hline \multicolumn{3}{|c|}{$\begin{array}{l}\text { QUADRO } X \text {. Resumo com as principais forças de } \\
\text { recomendação das técnicas de medição da PA do } \\
\text { ambulatório (AMPA e MAPA) em relação aos diferentes } \\
\text { parâmetros avaliados. }\end{array}$} \\
\hline & AMPA & MAPA \\
\hline - Identificação do risco CV & $\mathrm{B}^{*}$ & $A^{*}$ \\
\hline - Melhor controle da PA & $A^{* *}$ & $A^{* *}$ \\
\hline - Redução de gastos com HTA & $\mathrm{B} \ddagger$ & $B \ddagger$ \\
\hline
\end{tabular}

* MAPA permite identificação da PA nocturna, com evidência que este é o melhor parâmetro para a avaliação do risco

**Embora haja evidência convincente para a MAPA, a AMPA tem a vantagem de ser a técnica mais apropriada para uso repetido no controlo da PA, razão pela qual tem sido mais estudada neste desígnio.

CV.A evidência em relação à AMPA apenas provém ainda de estudos primários.

‡ Ambas têm evidência de reduzir custos com a HTA, mas AMPA é a técnica com menores custos.

cesso metodológico. A AMPA tem vantagens como técnica mais acessível e económica, permitindo ainda o acompanhamento a longo prazo do hipertenso e com o potencial de aumentar a adesão e comprometimento deste no seu tratamento. Por outro lado, a MAPA é técnica que permite a obtenção da maior quantidade de informação, nomeadamente a PA nocturna.

Face à evidência actual em relação à utilidade da AMPA para o diagnóstico e seguimento do hipertenso, a sua acessibilidade e custo reduzido, as vantagens na capacitação dos doentes e inerente cumprimento terapêutico, e a preferência destes em relação a este método para um uso repetido, os autores reforçam a recomendação das sociedades europeia e americana de hipertensão no sentido da aplicação generalizada deste método na avaliação do doente hipertenso. Perante a evidência existente para a população portuguesa, deverão ser seguidas as recomendações europeias: a média de uma semana de medições, nas condições ideais de medição, duas medições de manhã em jejum, antes da medicação, e duas medições antes do jantar. Deverão ser excluídos os registos do primeiro dia. O cut-off diagnóstico de 135/85 mmHg deverá ser usado para o diagnóstico de hipertensão, correspondendo aos 140/90 mmHg da medição no consultório.

Os autores concordam ainda com a tendência científica crescente de explorar a viabilidade da disponibi- lização da MAPA ao nível dos cuidados de saúde primários. Esta técnica poderá ser útil em casos seleccionados, nomeadamente, para valores de AMPA no limiar diagnóstico, entre os 125/75 mmHg e os 135/85 mmHg, para doentes que prefiram esta técnica ou aqueles que revelem ansiedade prejudicial com a utilização da AMPA. No caso da população portuguesa, será necessário um estudo de qualidade a nível nacional que calcule o custo-benefício a médio-longo prazo desta técnica, tendo em conta as estimativas da prevalência de hipertensão da bata branca, incidência de HTA e custos associados à aquisição dos aparelhos e formação dos profissionais de saúde.

\section{REFERÊNCIAS BIBLIOGRÁFICAS}

1. Parati G, Omboni S, Bilo G. Why is out-of-office blood pressure needed? Hypertension 2009 Aug; 54 (2): 181-7.

2. Pickering TG, James GD, Boddie C, Harshfield GA, Blank S, Laragh JH. How common is white coat hypertension? JAMA 1988 Jan 1; 259 (2): 225-8.

3. Pickering TG, Davidson K, Gerin W, Schhwartz JE. Masked hypertension. Hypertension 2002 Dec; 40 (6) :795-6.

4. O'Brien E. Ambulatory blood pressure measurement: the case for implementation in primary care. Hypertension 2008 Jun; 51 (6): 143541.

5. Izzo Jr JL, Sica DA, Black HR. Hypertension Primer: the Essentials of High Blood Pressure. 4th ed. Dallas, TX: American Heart Association; 2008.

6. Kaplan NM, Rode BD. Technique of blood pressure measurement in the diagnosis of hypertension. In: UpToDate; Sep 2009.

7. Katakam R, Brukamp K, Townsend R. What is the proper workup of a patient with hypertension? Cleve Clinic J Med 2008 Sep; 75 (9): $663-$ 71.

8. Parati G, Stergiou GS, Asmar R, Bilo G, de Leeuw P, Imai Y, et al. European Society of Hypertension guidelines for blood pressure monitoring at home: a Summary Report of the Second Internacional Consensus Conference on Home Blood Pressure Monitoring.J Hypertens 2008 Aug; 26 (8): 1505-26.

9. Pickering TG, Shimbo D, Haas D. Ambulatory blood-pressure monitoring. N Engl J Med 2006 Jun 1; 354 (22): 2368-74.

10. Myers MG, Godwin M, Dawes M, Kiss A, Tobe SW, Kaczorowski J. Measurement of blood pressure in the office: recognizing the problem and proposing the solution. Hypertension 2010 Feb; 55 (2): 195-200.

11. Perloff $D$, Sokolow $M$, Cowan R. The prognostic value of ambulatory blood pressures. JAMA 1983 May 27; 249 (20): 2792-8.

12. O'Brien E, Sheridan J, O'Malley K. Dippers and non-dippers. Lancet 1998 Aug 13; 2 (8607): 397.

13. Ebell, MH, Siwek J, Weiss BD, Woolf SH, Susman J, Ewingman B, et al. Strength of recommendation taxonomy (SORT): a patient-centered approach to grading evidence in the medical literature. Am Fam Physician. 2004 Feb 1; 69 (3): 548-56

14. Pickering TG, Miller NH, Ogedegbe G, Krakoff LR, Artinian NT, Goff D, et al. Call to action on use and reimbursement for home blood pressure monitoring: Executive Summary: a joint scientific statement from 
the American Heart Association, American Society of Hypertension, and Preventive Cardiovascular Nurses Association. Hypertension 2008 Jul; 52 (1): 1-9.

15. Pickering TG, Hall JE, Appel LJ, Falkner BE, Graves J, Hill MN, et al. Recommendations for blood pressure measurement in humans and experimental animals: Part 1: blood pressure measurement in humans: a statement for professionals from the Subcommittee of Professional and Public Education of the American Heart Association Council on High Blood Pressure Research. Hypertension 2005 Jan; 45 (1): 142-161.

16. Mancia G, De Backer G, Dominiczak A, Cifkova R, Fagard R, Germano G, et al. 2007 Guidelines for the Management of Arterial Hypertension: the Task Force for the Management of the European Society of Hypertension (ESH) and the European Society of Cardiology (ESC). Eur Heart J 2007 Jun; 28 (12): 1462-536.

17. Hansen TW, Kikuya M, Thijs L, Björklund-Bodegard K, Kuznetsova T, Ohkubo T, et al. Prognostic superiority of daytime ambulatory over conventional blood pressure in four populations: a meta-analysis of 7030 individuals. J Hypertens 2007 Aug; 25 (8): 1554-64.

18. Conen D, Bamberg F. Noninvasive 24-h ambulatory blood pressure and cardiovascular disease: a systematic review and meta-analysis. J Hypertens 2008 Jul; 26 (7): 1290-9.

19. Fagard RH, Celis H, Thijs L, Staessen JA, Clement DL, de Buyzere ML, et al. Daytime and nighttime blood pressure as predictors of death and cause-specific cardiovascular events in hypertension. Hypertension 2008 Jan; 51 (1): 55-61.

20. Boggia J, Li Y, Thijs L, Hansen TW, Kikuya M, Björklund-Bodegard K, et al. International Database on Ambulatory Blood Pressure Monitoring in relation to Cardiovascular Outcomes (IDACO) investigators. Prognostic accuracy of day versus night ambulatory blood pressure: a cohort study. Lancet 2007 Oct 6; 370 (9594): 1219-29.

21. Brobie G, Chatellier G, Genes N, Clerson P, Vaur L, Vaisse B, et al. Cardiovascular prognosis of "masked hypertension" detected by blood pressure self-measurement in elderly treated hypertensive patients. JAMA 2004 Mar 17; 291 (11): 1342-9.

22. Asayama K, Ohkubo T, Kikuya M, Metoki H, Hoshi H, Hashimoto J, et al. Prediction of stroke by self-measurement of blood pressure at home versus casual screening blood pressure measurement in relation to the Joint National Committee 7 Classification - the Ohasama study. Stroke 2004 Oct; 35 (10): 2356-61.

23. Fagard RH, Van Den Broeke C, De Cort P. Prognostic significance of blood pressure measured in the office, at home and during ambulatory monitoring in older patients in general practice. J Hum Hypertens 2005 Oct; 19 (10): 801-7.

24. Sega R, Facchetti R, Bombelli M, Cesana G, Corrao G, Grassi G, et al. Prognostic value of ambulatory and home blood pressures compared with office blood pressure in the general population: follow-up results from the Pressioni Arteriose Monitorate e Loro Associazioni (PAMELA) study. Circulation 2005 Apr 12; 111 (14): 1777-83.

25. Stergiou GS, Baibas NM, Kalogeropoulos PG. Cardiovascular risk prediction based on home blood pressure measurement: the Didima study. J Hypertens 2007 Aug; 25 (8): 1590-6.

26. Shimada K, Fujita T, Ito S, Naritomi H, Ohihara T, Shimamoto K, et al. The importance of home blood pressure measurement for preventing stroke and cardiovascular disease in hypertensive patients: a sub-analysis of the Japan Hypertension Evaluation with Angiotensin II Antagonist Losartan Therapy (J-HEALTH) study, a prospective nationwide observational study. Hypertens Res 2008 Oct; 31 (10): 1903-11.
27. Niiranen TJ, Hänninen MR, Johansson J, Reunanen A, Jula AM. Homemeasured blood pressure is a stronger predictor of cardiovascular risk than office blood pressure - the FINN-HOME study. Hypertension 2010 Jun; 55 (6): 1346-51.

28. Eguchi K, Pickering TG, Hoshide S, Ishikawa J, Ishikawa S, Schwartz JE, et al. Ambulatory blood pressure is a better marker than clinic blood pressure in predicting cardiovascular events in patients with/without type 2 diabetes. Am J Hypertens 2008 Apr; 21 (4): 443-50.

29. Burr ML, Dolan E, O'Brien EW, O'Brien ET, McCormack P. The value of ambulatory blood pressure in older adults: the Dublin outcome study. Age Ageing 2008 Mar; 37 (2): 201-6.

30. de la Sierra A, Redon J, Banegas JR, Segura J, Parati G, Gorostidi M, et al; Spanish Society of Hypertension Ambulatory Blood Pressure Monitoring Registry Investigators. Prevalence and factors associated with circadian blood pressure patterns in hypertensive patients. Hypertension 2009; 53 Mar; 53 (3): 466-72.

31. Bastos JM, Bertoquini S, Polónia J. Cardiovascular prognostic value of ambulatory blood pressure monitoring in a Portuguese hypertensive population followed up for 8.2 years. Blood Press Mon 2010 Oct; 15 (5): 240-6.

32. Verberk WJ, Kroon AA, Kessels AG, Leeuw PW. Home blood pressure measurement: a systematic review. J Am Coll Cardiol 2005 Sep 6; 46 (5): 743-51.

33. Schroeder K, Fahey T, Ebrahim S. Interventions for improving adherence to treatment in patients with high blood pressure in ambulatory settings. Cochrane Database Syst Reviews 2004; (2): CD004804.

34. Cappuccio FP, Kerry SM, Forbes L, Donald A. Blood pressure control by home monitoring: meta-analysis of randomised trials. BMJ 2004 Jul 17; 329 (7458): 145.

35. Bray EP, Holder R, Mant J, Mcmanus RJ. Does self-monitoring reduce blood pressure? Meta-analysis with meta-regression of randomized controlled trials. Ann Med 2010 Jul; 42 (5): 371-86.

36. Agarwal R, Bills JE, Hecht TJ, Light RP. Role of home blood pressure monitoring in overcoming therapeutic inertia and improving hypertension control: a systematic review and meta-analysis. Hypertension 2011 Jan; 57 (1): 29-38.

37. Ishikawa J, Carroll DJ, Kuruvilla S, Schwartz JE, Pickering TG. Changes in home versus clinic blood pressure with antihypertensive treatments: a meta-analysis. Hypertension 2008 Nov; 52 (5): 856-64.

38. Mancia G, Parati G. Office compared with ambulatory blood pressure in assessing response to antihypertensive treatment: a meta-analysis. J Hypertens 2004 Mar; 22 (3): 435-45.

39. Maldonado J, Pereira T; Estudo AMPA. Auto-medição da pressão arterial na hipertensão arterial - análise preliminar do estudo AMPA. Rev Port Cardiol 2009 Jan; 28 (1): 7-21.

40. Obara T, Ohkubo T, Asayama K, Metoki H, Inoue R, Kikuya M, et al. Home blood pressure measurements associated with better blood pressure control: the J-HOME study. J Hum Hypertens 2008 Mar; 22 (3): 197204.

41. McManus R, Mant J, Bray EP, Holder R, Jones MI, Greenfield S, et al. Telemonitoring and self-management in the control of hypertension (TASMINH2): a randomized controlled trial. Lancet 2010 Jul 17; 376 (9736): 163-72.

42. Manning G, Brooks A, Slinn B, Millar-Craig MW, Donnelly R. Assessing blood pressure control in patients treated for hypertension: comparing different measurements and targets. Br J Gen Pract 2006 May; 56 (526):375-7. 
43. Banegas JR, Segura J, Sobrino J, Rodríguez-Artalejo F, de la Sierra A, de la Cruz JJ, et al. Effectiveness of blood pressure control outside the medical setting. Hypertension 2007 Jan; 49 (1): 62-8.

44. Conen D, Tschudi P, Martina P. Twenty-four hour ambulatory blood pressure for the management of antihypertensive treatment: a randomized controlled trial. J Hum Hypertens 2009 Feb; 23 (2):122-9.

45. Zaninelli A, Parati G, Cricelli C, Bignamini AA, Modesti PA, Pamparana F, et al. Office and 24-h ambulatory blood pressure control by treatment in general practice: the 'Monitoraggio della pressione ARteriosa nella medicina TErritoriale' study. J Hypertens 2010 May; 28 (5): 910-7.

46. Fukunaga H, Ohkubo T, Kobayashi M, Tamaki Y, Kikuya M, Obara T, et al. Cost-effectiveness of the introduction of home blood pressure measurement in patients with office hypertension. J Hypertens 2008 Apr; 26 (4): 685-90.

47. Krakoff LR. Cost-effectiveness of ambulatory blood pressure: a reanalysis. Hypertension 2006 Jan; 47 (1): 29-34.

48. Ewald B, Pekarsky B. Cost analysis of ambulatory blood pressure monitoring in initiating antihypertensive drug treatment in Australian general practice. Med J Aust 2002 Jun 17; 176 (12): 580-3.

49. Lorgelly P, Siatis I, Brooks A, Slinn B, Millar-Craig MW, Donnelly R, et al. Is ambulatory blood pressure monitoring cost-effective in the routine surveillance of treated hypertensive patients in primary care? $\mathrm{Br} J \mathrm{Gen}$ Pract 2003 Oct; 53 (495): 794-6.

50. Pessanha P, Viana M, Ferreira P, Bertoquini S, Polónia J. Valor diagnóstico e análise custo-benefício da monitorização ambulatória da pressão arterial de 24 horas (MAPA) em Cuidados de Saúde Primários no Centro de Saúde de São João - Porto (sumário), 2008. Disponível em http://news.fm.ul.pt/Backoffice/UserFiles/File/News6files/(Resumo_Mencao\%20Honrosa\%20Centro\%20Saude\%20S.\%20Joao.pdf [acedido em 13/07/2011].

\section{CONFLITOS DE INTERESSE}

Os autores declaram não possuir conflitos de interesse nem financiamento do estudo.

\author{
ENDEREÇO PARA CORRESPONDÊNCIA \\ Hélder Teixeira Aguiar \\ Rua Guerra Junqueiro 241 \\ 3700-132 S. João da Madeira \\ Email:Aguiar.HelderG@gmail.com
}

Recebido em 25/10/2010

Aceite para publicação em 22/06/2011

\section{ABSTRACT}

\section{AMBULATORY EVALUATION OF BLOOD PRESSURE: AN EVIDENCE-BASED REVIEW}

Objectives: To assess the evidence for the usefulness of self-measurement of blood pressure (SMBP) and ambulatory blood pressure monitoring (ABPM) as part of the evaluation and follow-up of hypertensive individuals in the prognosis of cardiovascular disease, blood pressure control and cost-effectiveness compared with isolated office blood pressure monitoring (OBPM) and to assess the prognostic value of daytime and night time blood pressure (BP), and the day/night blood pressure ratio, as measured by ABPM.

Data Sources: Medline and Evidence-Based Medicine databases.

Review methods: A survey of clinical guidelines, systematic reviews, meta-analysis, clinical trials and observational studies, published between January 2000 and May 2011 was performed using the MeSH term "Blood Pressure Monitoring, Ambulatory" and the key words "Self Blood Pressure Monitoring" and "Home Blood Pressure Monitoring". The SORT scale of the American Family Physician was applied to grade the evidence.

Conclusions: Thirty eight articles were selected for analysis. These included four clinical practice guidelines, two systematic reviews, nine meta-analysis, 19 clinical trials and four observational studies. Each of the ambulatory blood pressure measurements studied provides a reduction in the white coat effect, with not only a stronger association with cardiovascular events, but also a higher degree of control of blood pressure. Ambulatory evaluation of blood pressure also reduces costs through the reduction in the costs of medication and other treatments.

For each of the ambulatory blood pressure measurement techniques studied, there is evidence that their use can provide a more accurate diagnostic and prognostic evaluation (strength of recommendation A for ABPM and strength of recommendation $B$ for SBPM). These methods may result in better control of blood pressure in the hypertensive patient (strength of recommendation $A$ for both ABPM and SBPM). Ambulatory measurement may also reduce costs (strength of recommendation $B$ for both ABPM and SBPM).

Key-words: Hypertension; Blood Pressure Monitoring, Ambulatory; Self Blood Pressure Monitoring; Home Blood Pressure Monitoring. 